\title{
Determination of Wavelet Ridges of Nonstationary Signals by Singular Value Decomposition
}

\author{
Nalan Özkurt and F. Acar Savacı
}

\begin{abstract}
The ridges obtained from chaotic signals can give the relevant information about the phase structures of the dynamical systems. Therefore, a new wavelet ridge determination method for the noisy signals and nonstationary signals, which is based on the singular value decomposition (SVD) has been proposed in this paper. The proposed method has been compared with Carmona method for monocomponent signals, and multicomponent signals. The proposed method is computationally more effective than the Carmona method to determine the actual ridges. Also, the ridges of the periodic limit cycles and chaotic attractors have been determined by using the SVD-based method to find the degree of chaoticity.
\end{abstract}

Index Terms-Instantaneous frequency, singular value decomposition (SVD), wavelet ridge.

\section{INTRODUCTION}

D UE TO THE nonstationary property of most of the signals from nature, the study of nonstationary signals in many different fields of science and engineering is very important and the wavelet analysis has found a wide interest in various areas such as in mechanics [1], in data compression [2], in molecular dynamics [3], in geophysics [4], in biomedical engineering [5], [6].

Because of the nonstationary nature of the chaotic waveforms, the chaotic waveforms have also been analyzed in the time-frequency domain especially by Wigner distribution [7], [8] and by wavelets [9]-[12]. The time-frequency representation of the trajectories would be more convenient to get dynamical information about the chaotic system, since the asymptotic quantities (Lyapunov exponents, entropy, fractal dimension) only reflect the asymptotical behavior. This representation can reveal the phase-space structures by obtaining the relevant information from a single trajectory through the extraction of main frequencies along the ridge (i.e., a curve at the time-frequency plane along which the energy is locally maximum). The original signal can be recovered using the skeleton of the transform [13]-[15] which is defined as the values of the wavelet transform (WT) coefficients along the ridges. The instantaneous frequencies which have been determined through the ridges can also be used to detect the resonance

Manuscript received August 8, 2003; revised March 2, 2004. This paper was recommended by Associate Editor W. X. Zheng.

N. Özkurt is with Dokuz Eylul University, Izmir, Turkey (e-mail: nalan.ozkurt@eee.deu.edu.tr).

F. A. Savacı is with Izmir Institute of Technology, Izmir, Turkey (e-mail: acarsavaci@iyte.edu.tr).

Digital Object Identifier 10.1109/TCSII.2005.849041 trappings and to characterize the degree of the chaoticity [9]. The ridge-detection algorithm based on the phase of the WT has been given in [16], [17]. The extension of this work to noisy mono-component and multicomponent signals have been done in [18] and [13], respectively. In this paper, the determination of the wavelet ridges of noisy signals and chaotic signals based on the singular value decomposition (SVD) has been introduced.

In Section II, after briefly introducing the WTs, the scalogram matrix has been constructed. In Section III, the definitions of the analytical signal, the instantaneous frequency and the ridge of the WT have been given. The proposed method based on SVD to determine the ridges has been introduced in Section IV and the given method has been applied in Section $\mathrm{V}$ to the chirp signals and chaotic signals arising from Chua's circuit [19].

\section{WAVELET ANALYSIS}

The wavelets are the family of the signals that is produced by the translations and the dilations of a mother wavelet satisfying the admissibility condition. The continuous WT coefficients of a signal $s(t) \in L^{2}(\mathbb{R})$ are determined for the different scales and translations as follows:

$$
W_{s}(a, b ; \Psi) \triangleq \int_{-\infty}^{\infty} s(t) \Psi_{a, b}^{*}(t) d t
$$

where $a$ and $b$ are the dilation (scale) and translation coefficients, respectively, and $*$ denotes the complex conjugate; the scaled and translated wavelet is obtained as

$$
\Psi_{a, b}(t) \triangleq \frac{1}{a} \Psi\left(\frac{t-b}{a}\right), \quad a \in \mathbb{R}^{+} ; \quad b \in \mathbb{R}
$$

where $\Psi(\cdot)$ is the mother wavelet. The mother wavelet must satisfy the admissibility condition which implies zero mean as

$$
0<c_{\psi} \triangleq \int_{0}^{\infty}|\hat{\Psi}(\omega)|^{2} \frac{d \omega}{|\omega|}<\infty
$$

as given in [5], where $\hat{\Psi}(\omega)$ is the Fourier transform of the mother wavelet.

A local time-frequency energy density called scalogram $P_{s}(a, b ; \Psi)$ has been defined in the wavelet domain as [20]

$$
P_{s}(a, b ; \Psi) \triangleq\left|W_{s}(a, b ; \Psi)\right|^{2} .
$$

The continuous WT conserves the total energy of the signal $E_{T}$ according to the Plancherel's formula as in [16]

$$
\begin{aligned}
E_{T} \triangleq\|s\|_{2}^{2} & =\int_{-\infty}^{\infty}|s(t)|^{2} d t \\
& =c_{\psi}^{-1} \int_{-\infty}^{\infty} \int_{0}^{\infty}\left|W_{s}(a, b ; \Psi)\right|^{2} \frac{d a d b}{a} .
\end{aligned}
$$


For the numerical computations, the discrete samples of the continuous WT have been considered and the scaled and translated wavelets have been defined at the dyadic grid as

$$
\Psi_{m n}(t)=a_{0}^{-m / 2} \Psi\left(a_{0}^{-m} t-n b_{0}\right) \quad m, n \in \mathbb{Z}
$$

where $a_{0}, b_{0} \in \mathbb{R}$ are the dilation and translation coefficients, respectively. Then, the numerical approximation of the total energy gives

$$
E_{T} \approx \frac{k}{c_{\psi}} \sum_{m=-\infty}^{\infty} \sum_{n=-\infty}^{\infty}\left|c_{m n}\right|^{2}
$$

with $k \triangleq b_{0} \ln a_{0}$ and WT coefficients are defined as

$$
c_{m n} \triangleq W_{s}\left(a_{m}, b_{n} ; \Psi\right)
$$

where $a_{m} \triangleq a_{0}^{-m}, b_{n} \triangleq n b_{0}$.

If the wavelets defined in (6) are chosen such as to constitute a Riesz basis for every $s(t) \in L^{2}(\mathbb{R})$ then the approximate energy is bounded with the energy bounds which can be calculated from the frame bounds defined in [20].

By considering only finite number of basis vectors for practical purposes, the approximate total energy in (7) can be further approximated as

$$
\tilde{E}_{T} \triangleq \frac{k}{c_{\psi}} \sum_{m=1}^{M} \sum_{n=1}^{N} p_{m n}
$$

where the entries of the scalogram matrix $P=\left[p_{m n}\right]_{M \times N}$ are defined as

$$
p_{m n} \triangleq\left|c_{m n}\right|^{2}
$$

which are the local time-frequency domain energy densities evaluated at the discrete dilations $a_{m}$ and translations $b_{n}$.

The truncation of the infinite dimensional scalogram matrix in time has been done by observing the attractor of the chaotic signal in the phase space.

For the nonstationary signals with compact support, only the samples in the support have been used to construct the finite dimensional scalogram matrix. The truncation in frequency has been done when the wavelet coefficients were sufficiently small.

In this paper, the WT has been demonstrated by choosing the following standard complex Morlet mother wavelet

$$
\psi(t)=\frac{1}{\sqrt{2 \pi}} \cdot e^{j \omega_{0} t} e^{-t^{2} / 2}
$$

where $\omega_{0}$ defines the center frequency of the wavelet.

\section{WAVELET RIDGES}

In this section, before introducing the SVD-based ridge determination method, the related definitions will be given as follows.

Definition 1: The analytical mono-component signal $Z_{s}(t)$ associated with the real signal $s(t)$ is a complex function of time defined as

$$
Z_{s}(t) \triangleq s(t)+j \tilde{s}(t)=A(t) \exp (j \phi(t))
$$

where the function $\tilde{s}(t)$ is the Hilbert transform of $s(t)$ which is defined in [16]
Definition 2: The instantaneous angular frequency is defined as the derivative of the phase $\phi(t)$

$$
\omega_{\text {inst }}(t)=\frac{d \phi(t)}{d t}
$$

Definition 3: Let $s(t) \in L^{2}(\mathbb{R})$ be a real finite energy signal. The signal $s(t)$ is asymptotic iff

$$
\left|\frac{d \phi(t)}{d t}\right| \gg\left|\frac{1}{A(t)} \frac{d A(t)}{d t}\right|
$$

where $A(t)>0$ and $\phi(t) \in[0,2 \pi] \forall t \in \mathbb{R}$

For the chosen asymptotic mother wavelet in the form of $\Psi(t)=A_{\Psi}(t) e^{j \phi_{\Psi}(t)}$, the WT of the analytical signal $Z_{s}(t)$ can be found by using (1) and the integral WT of the real signal $s(t)$ is obtained as

$$
W_{s}(a, b ; \Psi)=\frac{1}{2 a} \int_{-\infty}^{\infty} A(t) A_{\Psi}\left(\frac{t-b}{a}\right) \exp \left(j \Phi_{a, b}(t)\right) d t
$$

where $\Phi_{a, b}(t) \triangleq \phi(t)-\phi_{\Psi}((t-b) / a)$ [20]. Since the integrand is asymptotic, the stationary phase theorem given in [16] and [17] can be directly applied.

Let $t=t_{0}$ be the stationary point of the argument of the integrand

$$
\begin{aligned}
& \Phi_{a, b}^{\prime}\left(t_{0}\right)=\phi^{\prime}\left(t_{0}\right)-\frac{1}{a} \quad \phi_{\Psi}^{\prime}\left(\frac{t_{0}-b}{a}\right)=0 \\
& \Phi_{a, b}^{\prime \prime}\left(t_{0}\right)=\phi^{\prime \prime}\left(t_{0}\right)-\frac{1}{a^{2}} \phi_{\Psi}^{\prime \prime}\left(\frac{t_{0}-b}{a}\right) \neq 0
\end{aligned}
$$

[21], and then the WT in (15) can be approximated as in [17] by

$$
W_{s}(a, b ; \Psi) \approx \sqrt{\frac{\pi}{2}} \frac{Z_{s}\left(t_{0}\right) \Psi^{*}\left(\frac{t_{0}-b}{a}\right)}{\operatorname{corr}(a, b)}
$$

where

$$
\operatorname{corr}(a, b)=a\left|\Phi_{a, b}^{\prime \prime}\left(t_{0}\right)\right|^{1 / 2} e^{-j(\pi / 4) \operatorname{sgn}\left[\Phi_{a, b}^{\prime \prime}\left(t_{0}\right)\right]} .
$$

Since at $t_{0}=b$, the chosen translated and dilated Morlet mother wavelet in (18) has its maximum, then at that time instant $W_{s}(a, b ; \Psi)$ is also locally maximum neglecting the correction term.

Definition 4: The ridge of the WT of $s(t)$ is the set of points $(a, b)$ which are the stationary points of the argument of the WT " $\Phi_{a, b}(t)$ " in (15) (i.e., $t_{0}(a, b)=b$ ).

The ridge can be obtained from (16) as

$$
a(b)=\frac{\phi_{\Psi}^{\prime}(0)}{\phi^{\prime}(b)}
$$

and then the instantaneous angular frequency along the ridge becomes

$$
\left.\omega_{\text {inst }}(t)\right|_{t=b}=\phi^{\prime}(b)=\frac{\phi_{\Psi}^{\prime}(0)}{a(b)} .
$$

The multicomponent signal with the instantaneous amplitudes $A_{l}(t) \in \mathcal{C}^{1}$ and the instantaneous phases $\phi_{l} \in \mathcal{C}^{2}$ can be described by

$$
s(t)=\sum_{l=1}^{L} A_{l}(t) e^{j \phi_{l}(t)}
$$

where $L$ is the number of the components. 
The WT of the multicomponent signal $s(t)$ has been given in [13] as

$$
W_{s}(a, b ; \Psi)=\frac{1}{2} \sum_{l=1}^{L} A_{l}(b) e^{j \phi_{l}(b)} \hat{\Psi}^{*}\left(a \phi_{l}^{\prime}(b)\right)+r(a, b)
$$

where $r(a, b) \sim O\left(\left|A_{l}^{\prime}\right|,\left|\phi_{l}^{\prime \prime}\right|\right)$.

If the Fourier transform of the mother wavelet " $\hat{\Psi}(\omega)$ " is localized near a certain angular frequency $\omega=\omega_{0}$, the scalogram is localized around $L$ ridges

$$
a^{l}=a^{l}(b)=\frac{\omega_{0}}{\phi_{l}^{\prime}(b)}, \quad l=1, \ldots, L .
$$

The representation above is valid at $t=b$ if the instantaneous frequencies of the components of the signal are separable [20].

\section{SVD-BASED RIDGE DETERMINATION}

In the literature, there are several ridge-detection methods such as stationary phase method which calculates the ridge using the stationary point theorem [16], [17]; the simple method which directly determines the local maxima of the scalogram [9], [17]; the Carmona method which converts the detection problem into an optimization problem [18] and its extension to the multicomponent signals using the algorithm based on the Markov Chain Monte Carlo method [13]. In this section, the wavelet ridge-detection algorithm based on singular value decomposition of the scalogram of the mono-component and multicomponent nonstationary signals in the presence of noise and measurement errors has been explained. The proposed algorithm is different than the ones given in [13], [22] which apply SVD directly to the data matrix, but it is similar to the algorithms which apply the SVD method to the WT coefficient matrix [23], [24]. In [23], the first left singular vector called as pseudo power signature is used in the classification of the seismic signals. The left and right singular vectors are used to characterize the speech signals in [24].

Lemma 1: The Frobenius norm of an $M \times N$ matrix $A$ of rank $K$

$$
E_{A}=\|A\|_{F}^{2}=\sum_{i=1}^{M} \sum_{j=1}^{N}\left|a_{i j}\right|^{2}=\sum_{k=1}^{K} \sigma_{k}^{2}
$$

where $\sigma_{k}$ are the singular values of $A$ [25], [26].

The lemma states that the energy of the signal in wavelet domain is related with the singular values. Since the singular values are in decreasing order, the most of the energy is contained in the components associated with the greater singular values.

The scalogram matrix $P \in \mathbb{R}^{M \times N}$ with $\operatorname{rank}(P)=K$ can be decomposed into its singular values as

$$
P=U \Sigma V^{T}
$$

where the singular value matrix $\Sigma \in \mathbb{R}^{M \times N}$ the orthogonal matrices $U \in \mathbb{R}^{M \times M}$ and $V \in \mathbb{R}^{N \times N}$ and these matrices can be decomposed into two parts

$$
\begin{aligned}
\Sigma & =\left[\begin{array}{cc}
\Sigma_{s} & \theta \\
\theta & \Sigma_{n}
\end{array}\right] \\
U & =\left[\begin{array}{ll}
U_{s} & U_{n}
\end{array}\right] \\
V & =\left[\begin{array}{ll}
V_{s} & V_{n}
\end{array}\right]
\end{aligned}
$$

where the $\mathbf{U}_{\mathbf{s}} \in \mathbb{R}^{M \times S}, \Sigma_{\mathbf{s}} \in \mathbb{R}^{S \times S}$, and $\mathbf{V}_{\mathbf{s}} \in \mathbb{R}^{N \times S}$ where $S \leq K$ representing the dominant components associated with the original signal, and the less significant components and the noise are included in $\boldsymbol{\Sigma}_{\mathbf{n}} \in \mathbb{R}^{M-S \times N-S}, \mathbf{U}_{\mathbf{n}} \in \mathbb{R}^{M \times M-S}$, and $\mathbf{V}_{\mathbf{n}} \in \mathbb{R}^{N \times N-S}$.

The effects of the additive white Gaussian noise (AWGN) is higher on the smaller singular values which correspond to the components of the signal with lower energy levels when SNR is not too low. Therefore, the effect of the noise is reduced by truncating the lower singular values. The approximated scalogram matrix $\tilde{P}$ with rank $S$ is obtained as

$$
\tilde{P}=U_{s} \Sigma_{s} V_{s}^{T}
$$

by using only the signal components $U_{s}, \Sigma_{s}$, and $V_{s}$.

The number of the singular values included in approximation $\Sigma_{s}$ has been defined by considering the ratio of the energy associated with the singular values to the total energy

$$
r_{k} \triangleq \frac{\sigma_{k}^{2}}{E_{T}}
$$

where $E_{T}$ is the total energy of the scalogram matrix. For the given noise threshold $\varepsilon(0<\varepsilon \leq 1)$, the singular values with $r_{1} \geq r_{2} \geq \ldots \geq r_{S} \geq \varepsilon$ have been included in $\Sigma_{s}$ and the remaining $K-S$ singular values have been considered as the noise or the minor energy components.

In the wavelet domain at the fixed time instant $b_{j}$ the maximum energy occurs at the scale $a_{j}^{\text {main }}$, i.e.,

$$
\tilde{p}_{a_{j}^{\text {main }}, j} \triangleq\left\|\tilde{p}_{j}\right\|_{\infty}
$$

where $\tilde{p}_{j} \in \mathbb{R}^{M}$ denotes the $j$ th column of the approximated scalogram matrix $\tilde{P}$ and

$$
a_{j}^{\text {main }} \triangleq a^{\text {main }}\left(b_{j}\right) \quad j=1, \ldots, N
$$

and $a^{\text {main }}(b)$ is the main ridge of the approximated scalogram. Therefore, the main ridge can be calculated by determining the global maximum (assuming it exists for most of the practical signals) of the approximated scalogram for each time instant $t=b_{j}$.

The ridges of the multicomponent signal with sufficiently large amount of energy are also taken into account because the energy contribution of these components can not be neglected. The ridges are defined as

$$
\begin{gathered}
a_{j}^{l}=a^{l}\left(b_{j}\right), \quad j=1, \ldots, N ; \quad l=1, \ldots, L \\
\tilde{p}_{a_{j}^{l}, j} \geq \mu \tilde{p}_{a_{j}^{\text {main }}, j}, \quad 0<\mu<1
\end{gathered}
$$

where $\mu$ is the energy threshold and then the ridges are determined by finding the local maxima of the approximated scalogram for each time instant. In the case of mono-component signal, since the signal has single instantaneous frequency at a given time instant, a single ridge (the main ridge) will be sufficient to locate the energy concentrations.

\section{APPLICATIONS}

In this section, some of the applications of the proposed method have been given.

The center angular frequency of the mother wavelet has been chosen as $\omega_{0}=2 \pi$ for the examples given below. 

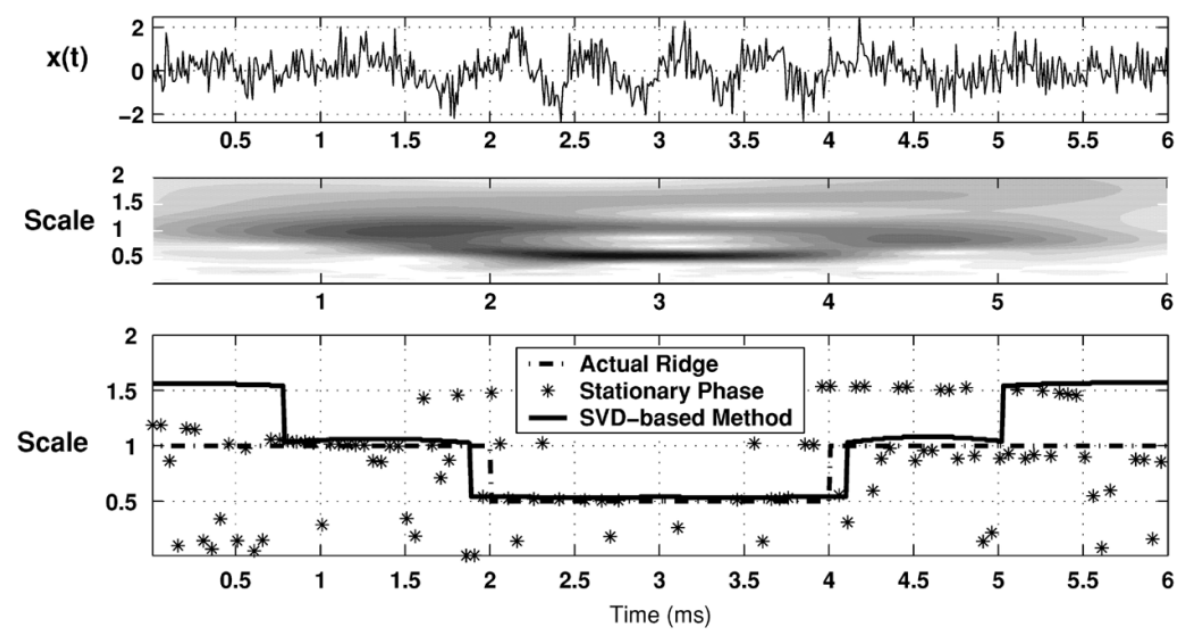

(b)

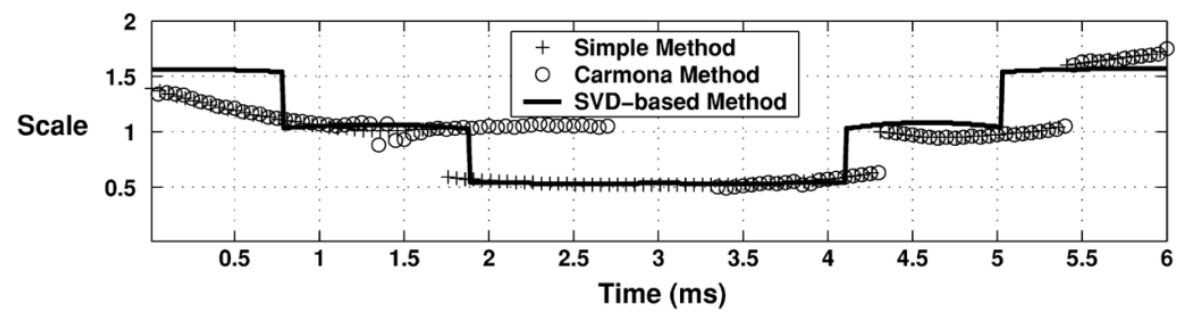

(d)

Fig. 1. (a) Time-waveform. (b) Scalogram. (c) Actual ridge, ridges calculated by stationary phase, and SVD-based method. (d) Ridges calculated by simple method, Carmona method and SVD-based method for chirp signal with additive Gaussian noise.
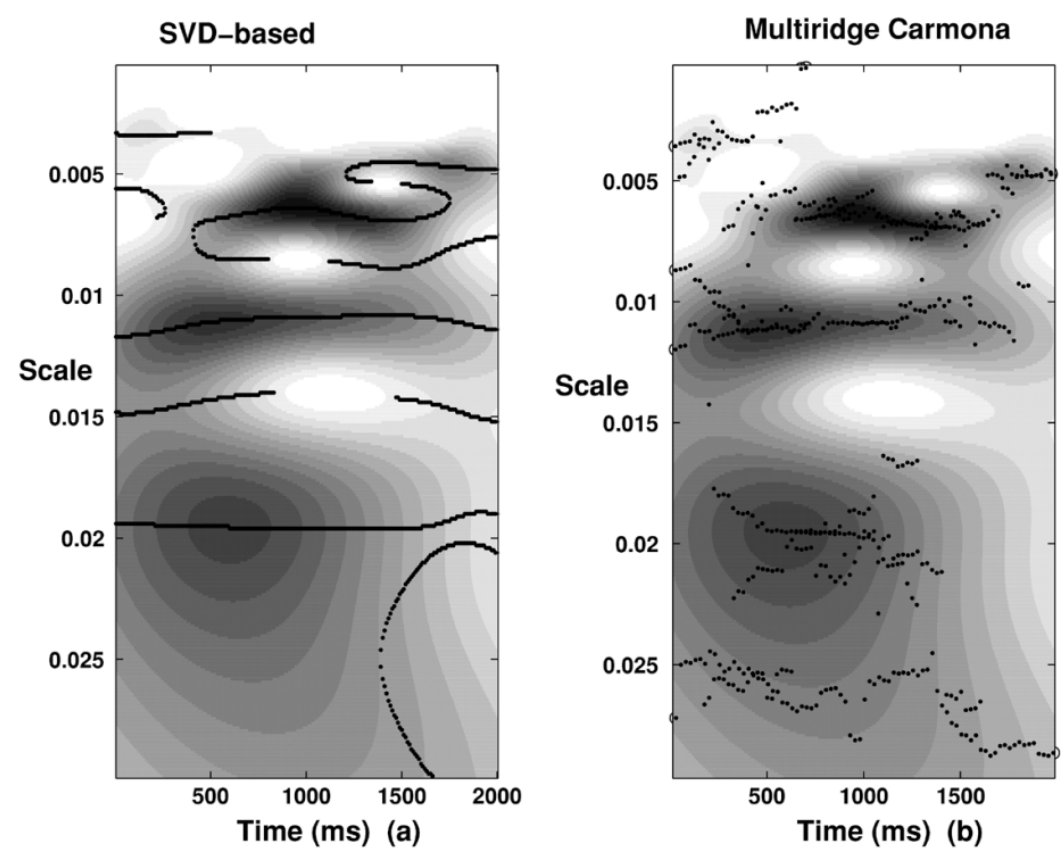

Fig. 2. Ridges on the scalogram of double-scroll attractor.

Example 1: The monocomponent chirp signal is given as follows:

$$
\begin{aligned}
& x(t)= \\
& \begin{cases}0.5\left(1-\cos \left(\frac{2 \pi t}{N \Delta t}\right)\right) \cos \left(2 \pi f_{0} t\right), & t \in[0,2] \cup(4,6] \\
0.5\left(1-\cos \left(\frac{2 \pi t}{N \Delta t}\right)\right) \cos \left(2 \pi 2 f_{0} t\right), & t \in(2,4] \\
0, & \text { elsewhere }\end{cases}
\end{aligned}
$$

where $f_{0}=1 \mathrm{~Hz}$ and $N$ is the number of samples and $\Delta t$ is the sampling time which are chosen as 600 and 0.01 , respectively.

For comparison of the methods, the chirp signal is embedded in Gaussian noise with signal to noise ratio SNR $=-3 \mathrm{~dB}$ and the scalogram matrix has been calculated for $M=40$ dilations which include the frequency range in which the energy of the signal is localized. The time waveform of the noisy chirp signal is shown in Fig. 1(a) and its scalogram is illustrated in Fig. 1(b). The actual ridge and the ridges calculated by 


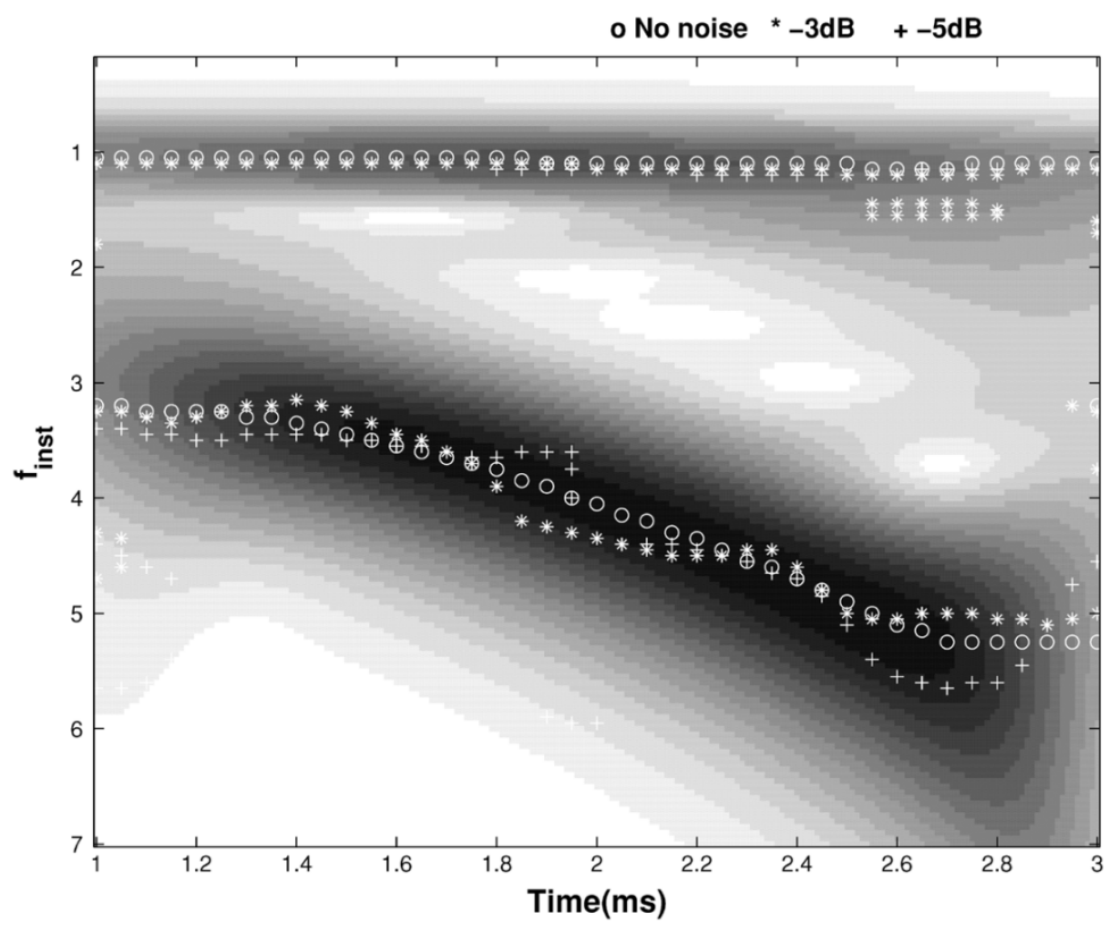

Fig. 3. Scalogram and the ridges of the signal with two hyperbolic chirps.

Period-1 Limit Cycle

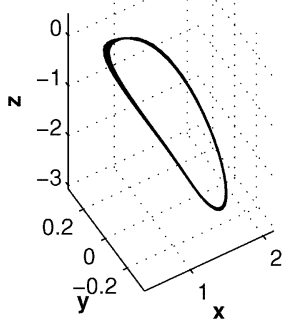

(a)

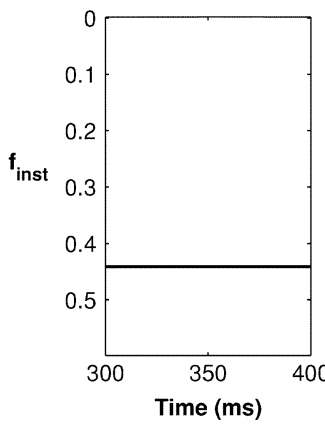

(e)
Quasiperiodic Attractor

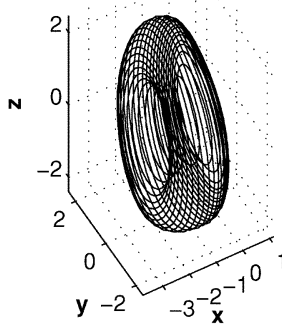

(b)

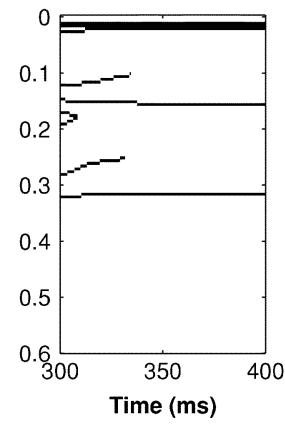

(f)
Spiral Attractor

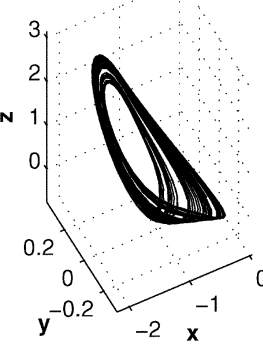

(c)

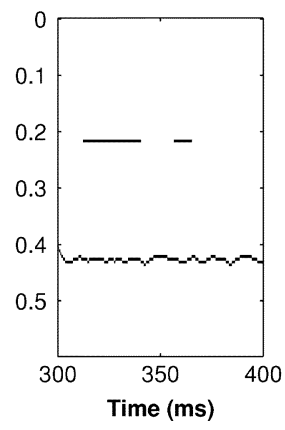

(g)
Double-Scroll Attractor

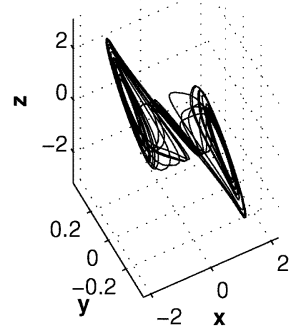

(d)

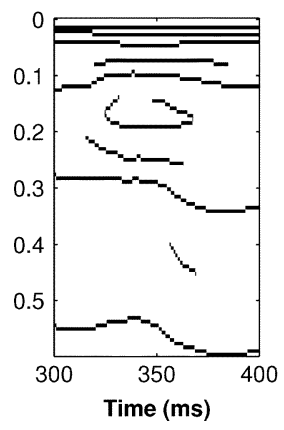

(h)

Fig. 4. Instantaneous frequencies of the signals from Chua's circuit by the SVD-based method.

Stationary Phase method are shown in Fig. 1(c), while ridges calculated by Simple method and Carmona method are shown in Fig. 1(d). The proposed method has been illustrated in both Figs. 1(c) and (d) for comparison purposes.

The ridge finding algorithms have been implemented using MATLAB in the PC with $1.4 \mathrm{GHz}$ CPU. The computation times of the algorithms for the stationary phase method, simple method, multiridge Carmona method and SVD-based method are $134.613 \mathrm{~s}, 0.030 \mathrm{~s}, 50.082 \mathrm{~s}$, and $3.715 \mathrm{~s}$, respectively.
Example 2: The wavelet ridges of the double-scroll attractor of Chua's circuit [27] has been determined by both multiridge Carmona method and the proposed method.

Four singular values of which energy contribution is greater than $10 \%$ of the total energy have been included in the approximated scalogram $\tilde{P}$. Then, the local maxima of the approximate scalogram $\tilde{P}$ has been obtained with energy threshold $\mu=0.1$. The ridges calculated by multiridge Carmona method and SVD-based method are shown in Fig. 2. Both methods pro- 
duce acceptable results showing the energy concentrations of the double-scroll attractor.

Both algorithms have been implemented using MATLAB and the computation times for multiridge Carmona method and SVD-based method are $1452.3 \mathrm{~s}$ and $22.12 \mathrm{~s}$, respectively. The computational cost of the Carmona method grows drastically when the size of the scalogram matrix becomes large, however the computations are faster when the ridges are calculated by SVD-based method.

Example 3: Performance of SVD-Based Method Under Additive White Gaussian Noise: The signal containing two hyperbolic chirps is defined as

$$
s(t)= \begin{cases}\sin \left(2 \pi \frac{\alpha_{1}}{\beta-t}\right)+\sin \left(2 \pi \frac{\alpha_{2}}{\beta-t}\right), & 1 \leq t \leq 3 \\ 0, & \text { elsewhere }\end{cases}
$$

where $\alpha_{1}=100, \alpha_{2}=25$, and $\beta=7$. The ridges of the given signal has been determined by using the SVD-based method for noise-free case and for two different signal to noise ratios. The scalogram of the noise-free signal and the computed ridges are shown in Fig. 3.

Example 4: Wavelet Ridges of Periodic, Quasi-Periodic, and Chaotic Signals: The wavelet ridges of the signals measured from the dynamical systems give information about the phase space structures of the system such as periodicity, quasi-periodicity, chaoticity. Also, the strength of chaos (i.e., the stronger chaos, the faster the divergence of the initially nearby trajectories and the larger the Lyapunov characteristic exponents [28]) can be characterized by the geometry and number of the ridges: weak chaos is characterized by one main connected ridge whereas strong chaos is characterized by multiple short ridges distributed over the time-frequency plane without an order [9]. The ridges of the signals obtained from Chua's circuit are shown in Fig. 4.

\section{CONCLUSION}

In this paper, the wavelet ridge determination algorithm based on the singular value decomposition of the scalogram of the nonstationary signals has been explained. The method has been applied for noisy mono-component chirp signal and the performance has been compared with stationary phase method, the simple method and the Carmona method. The SVD-based method has also been applied to the double-scroll attractor of the Chua's circuit in order to evaluate the performance for the multicomponent signals. The proposed method is computationally more effective than the Carmona method for monocomponent and multicomponent signals in determining the actual ridges in noisy situations. Furthermore, the wavelet ridges of the periodic, quasi-periodic and chaotic signals arising from the Chua's circuit have been determined and it is observed that the characterization of the signals can be made considering the ridges of the signal.

\section{REFERENCES}

[1] D. Newland, Random Vibrations, Spectral, and Wavelet Analysis. New York: Longman, 1993.
[2] M. Vetterli and J. Kovacevic, Wavelets and Subband Coding. Upper Saddle River, NJ: Prentice-Hall , 1995.

[3] A. Askar, A. Cetin, and H. Rabitz, "Wavelet transform for the analysis of molecular dynamics," J. Phys. Chem., vol. 100, pp. 19 165-19 173, 1996.

[4] E. Foufoula-Georgiou and P. Kumar, Wavelets in Geophysics. San Diego, CA: Academic, 1994.

[5] M. Akay, Time Frequency and Wavelets in Biomedical Signal Processing. New York: IEEE Press, 1997

[6] R. Carmona, W. Hwang, and R. Frostig, "Wavelet analysis for brainfunction imaging," IEEE Trans. Med. Imag., vol. 14, no. 3, pp. 556-564, Sep. 1995.

[7] P. Chen, "Study of chaotic dynamical systems via time-frequency analysis," in Proc. IEEE Int. Symp. Time-Frequency and Time-Scale Analysis, Oct. 1994, pp. 357-360.

[8] L. Galleani, M. Biey, M. Gilli, and L. Presti, "Analysis of chaotic signals in the time-frequency plane," in Proc. IEEE Int. Conf. Nonlinear Signal and Image Processing (NSIP'99), Antalya, Turkey, 1999, pp. 100-104.

[9] C. Chandre, S. Wiggins, and T. Uzer, "Time-frequency analysis of chaotic systems," Phys. D, vol. 181, pp. 171-196, 2003.

[10] N. Özkurt and F. Savacı, "Wavelet analysis of the generalized Chua's circuit," in Proc. 9th Workshop Nonlinear Dynamics of Electronic Sytems, Delft, The Netherlands, Jun. 2001, pp. 100-104.

[11] L. Wong and J. C. Chen, "Nonlinear and chaotic behavior of structural system investigated by wavelet transform techniques," Int. J. Nonlin. Mech., vol. 36, pp. 221-235, 2001.

[12] D. Allingham, M. West, and A. Mees, "Wavelet reconstruction of nonlinear dynamics," Int. J. Bifurc. Chaos, vol. 8, pp. 2191-2201, 1998.

[13] R. Carmona, W. Hwang, and B. Torresani, "Multiridge detection and time-frequency reconstruction," IEEE Trans. Signal Process., vol. 47, no. 2, pp. 480-492, Feb. 1999.

[14] T. Quatieri, Discrete-Time Speech Signal Processing: Principles and Practice. Upper Saddle River, NJ: Prentice-Hall, 2001.

[15] N. Özkurt and F. Savac1, "Reconstruction of nonstationary signals in wavelet domain based on singular value decomposition," in Proc. 11th Workshop Nonlinear Dynamics of Electronic Sytems (NDES'2003), vol. 1, Scuol, Switzerland, 2003, pp. 181-184.

[16] N. Delprat, B. Escudie, P. Guillemain, R. Kronland-Martinet, P. Tchamitchian, and B. Torresani, "Asymptotic wavelet and Gabor analysis: Extraction of instantaneous frequencies," IEEE Trans. Inf. Theory, vol. 38, pp. 644-664, 1992.

[17] M. Todorovska, "Estimation of instantaneous frequency of signals using the continuous wavelet transform," Univ. of Southern California, Los Angeles, Tech. Rep. CE 01-07, 2001.

[18] R. Carmona and W. Hwang, "Characterization of signals by the ridges of their wavelet transforms," IEEE Trans. Signal Process., vol. 45, no. 10, pp. 2586-2590, Oct. 1997.

[19] L. Chua, M. Komuro, and T. Matsumoto, "The double-scroll family," IEEE Trans. Circuits Syst., Fundam. Theory Appl., vol. 33, pp. 1073-1118, 1999.

[20] S. Mallat, A Wavelet Tour of Signal Processing. San Diego, CA: Academic, 1999.

[21] B. Boashash, "Estimating and interpreting the instantaneous frequency of a signal-Part 1: Fundamentals," Proc. IEEE, vol. 80, no. 4, pp. 520-538, Apr. 1992.

[22] B. Pilgram and W. Schappacher, "Estimation of the dominant singular values for SVD based noise reduction method," Int. J. Bifurc. Chaos, vol. 8, pp. 571-580, 1998.

[23] V. Venkatachalam and J. Aravena, "Nonstationary signal classification using pseudo power signatures: The matrix SVD approach," IEEE Trans. Circuits Syst. II, Analog Digit. Signal Process., vol. 46, no. 12, pp. 1497-1505, Dec. 1999.

[24] M. Casey, "Auditory group theory with applications to statistical basis methods for structured audio," Ph.D. dissertation, Sch. Architecture and Planning, MIT Media Lab., Cambridge, MA, 1998.

[25] B. de Moor, "Mathematical concepts and techniques for modeling of static and dynamic systems," Ph.D. dissertation, Dept. of Electrotechnics, Katholieke University, Leuven, Belgium, 1988.

[26] G. H. Golub and C. V. Loan, Matrix Computations. Baltimore, MD The Johns Hopkins Univ. Press, 1996.

[27] L. Aguirre, G. Rodrigues, and E. Mendes, "Nonlinear identification and cluster analysis of chaotic attractors from a real implementation of Chua's circuit," Int. J. Bifurc. Chaos, vol. 7, pp. 1411-1423, 1997.

[28] H. Kantz and T. Schreiber, Nonlinear Time Series Analysis. Cambridge, U.K.: Cambridge Univ. Press, 1999. 